\title{
Dynamics of FUE
}

\author{
Parsa Mohebi, MD, FISHRS I Encino, California, USA I| info@parsamohebi.com; Jeanette Straga, DO | \\ Encino, California, USA
}

\begin{abstract}
There are a number of basic principles that every hair transplant surgeon should know regardless of which FUE hair transplant technique he or she uses. In this article, we explore a number of common, and challenging, problems faced during the hair transplant process. An in-depth examination of the causes of these challenges leads to the discovery of solutions as well as to making the procedures more efficient and precise. The purpose of this article is to review the basic anatomic variations of FUE and the multiple steps involved, including skin immobilization, punch techniques, and scoring grafts.

We evaluate the impact of these variables on the FUE graft harvesting process and the challenges they may pose to even the most skilled surgeon. We will compare the different methods and discuss the techniques that ensure the surgeon can obtain the highest quality grafts every time. The consistent assessment of graft quality and continuous communication between the surgeon and staff are key elements to performing a successful FUE transplant. The constant evaluation also enables surgeons to fine-tune their approach with every graft harvesting attempt in order to minimize the risk of damaging the grafts.

Setting and following a standardized process for FUE hair transplant ensures that we get reproducible results in every procedure.
\end{abstract}

\section{NOMENCLATURE}

The FUE process consists of making a circular incision around the follicular unit (FU) with a hollow punch then extracting the $\mathrm{FU}$ from the donor area.

Scoring refers to cutting the tissue to the full desired depth prior to extraction.

Superficial scoring refers to cutting through superficial epidermis prior to full scoring. This is sometimes necessary as a separate step prior to full scoring as it will be discussed in manual graft extraction.

Extraction or pulling the graft refers to pulling out the scored FU to create a graft.

\section{ANATOMICAL CONSIDERATIONS Anchor system}

This complex system consists of multiple structures that hold the follicular group within the surrounding tissues. The anchoring system includes the epithelium and superficial dermis that surround the follicles, a sebaceous gland, the insertion of the arrector pili muscle, the connections between the connective tissue sheath (CTS), and the surrounding connective tissue.

The circular incision or scoring should be able to release the graft from its anchor system (Figure 1). This allows an easy and minimally traumatic extraction of the graft. The punch depth of the scoring incision by convention is set at $2.5 \mathrm{~mm}$. Adjustments are made during the graft testing phase that are crucial in obtaining optimal results as even a minimal alteration (as small as $0.1 \mathrm{~mm}$ ) can increase the number of grafts appearing depressed, rotated, or lost in the punch. For scalp hair, the punch depth varies between a shallow $1.2 \mathrm{~mm}$ to a maximum depth of $3.5 \mathrm{~mm}$.

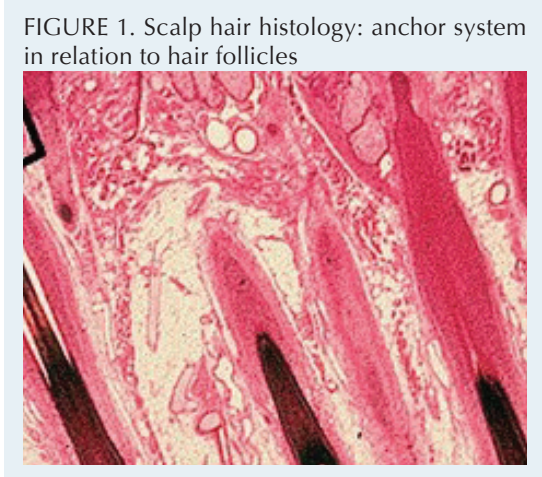

\section{Splay}

Follicles within each follicular unit don't always follow the same path along the entire length of their grouping. The distal (superficial) portion of the FU consists of a tightly bound bundle. Once beyond this bundle, the FU rests more freely within the tissue. At the very proximal (deep) aspect, which encompasses the dermal papillae, the individual hair follicles usually separate from each other (Figure 2). This unbound nature of the deep portion results in an outward splay of the bulb within the surrounding fat of deeper layers of the skin.

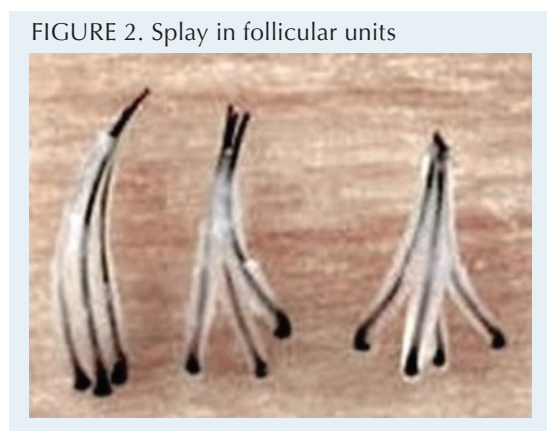

The splay of hair follicles is as important as the exit angles (discussed later). Because the hair follicles splay at the proximal levels, it is less likely that all will fit into the lumen of the punch. This tight bundle distally followed abruptly by the proximal outward splay increases the risk of transection of hair follicle. Fortunately, the percentage of FUs exhibiting significant splay is minimal as most hair follicles usually follow the same direction within their grouping as they dive deeper into the tissue. During FUE scoring, consideration is given to the minimal depth necessary to liberate the grafts from the anchor system.

Effect of splay on depth control. To avoid injury to the follicles, refinement of the depth of the excision is made by using depth control. Using graft testing, the depth control is tuned to the optimal depth to allow dislodgement of the anchoring system yet guard against overshooting depth resulting in transection (Figure 3).

Splay challenge. The challenge is when the splay occurs
FIGURE 3. Effect of splay on depth of punch in FUE

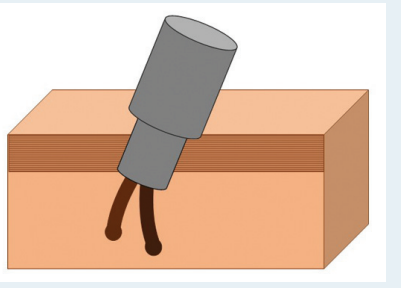


more superficially within or near the anchor system. When presented with this situation, we cut through the anchor system by slightly increasing the scoring punch depth, which invariably increases the transection rate.

Solution. The remedy to the high transection rate that results when the anchor system is too deep or when the splay starts superficially is to either increase the size of the punch or to minimize the sharpness of the punch. Increasing the size of the punch will enlarge the wound surface area while it may only minimally reduce the transection rate.

In contrast, reducing the sharpness of the punch can reduce the transection rate in most cases. One solution is the two-step extraction model that uses a sharp edge to initially superficially score the skin and a blunt edge to advance the dissection deeper. A working example of this duel cutting-edge method is the ARTAS ${ }^{\text {TM }}$ system, which artfully executes this punch within a punch technology. The S.A.F.E. system, by comparison, uses a rotating blunt punch tip as its cutting edge to minimize transection rate. The third option to avoid cutting the follicles with a sharp edge is a flat or

FIGURE 4. Splay challenge (Trumpet punch) with superficial splay and increased anchor depth

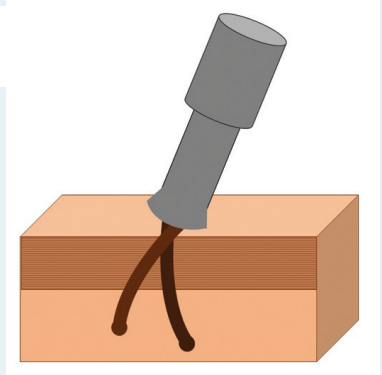
trumpet punch (Figure 4). Trumpet punches have a beveled sharp edge facing away from the center. A flat punch helps minimize the transection rate by creating a nominal deviation from the central axis of the FU. This edge is instrumental in reducing the transection rate especially when the operator chooses to increase the scoring depth to counteract the effect of a deep anchor system and/or exaggerated splay.

\section{Skin immobilization}

Immobilization of the skin is imperative for successful extraction of the grafts. A controlled method of multi-directional traction is applied to stabilize the donor skin for precise extraction of the grafts. There are several ways to provide the necessary traction. During a single case, a combination of the following methods may be used to facilitate extraction:

\section{- Three-point traction by scorer (single person).} While using the punch device, the index and middle fingers of the opposite hand pull against each

FIGURE 5. Three-point single operator traction: while using the punch device the index and middle fingers of the opposite hand pull against each other and against a portion of the hand that holds the punch.

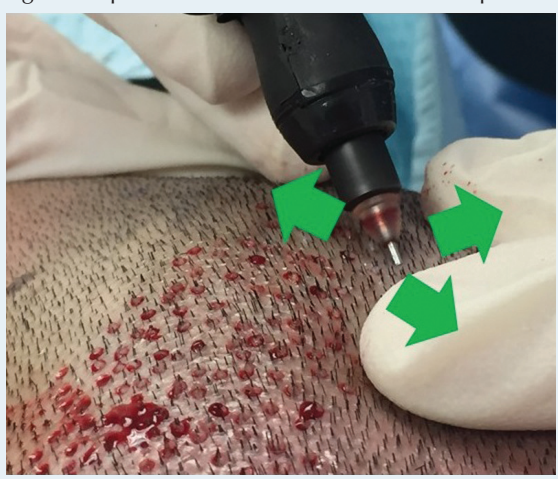

- Two-point traction (2 person).

The opposite hand of the operator provides upper traction while an assistant provides traction below, but near, the punch (Figure 6).

- Three-point traction (2 person). In addition to the operator's single-hand traction, two other hands can provide traction in opposing directions thus creating the taut field of 3-point traction (Figure 7).

\section{- Tumescent} effect. Superficial infiltration of the dermis with saline (with and without epinephrine) provides the best traction of the scalp (Figure 8). The tension of the tumescent effect lasts for a short time before the fluid dissipates into the interstitial space. Infiltration should be done in an orother and against portions of the hand that holds the punch. This creates a 3-point traction near where the operator is scoring. This method requires a certain amount of manual dexterity, but if done properly, it is one of the most effective methods of skin immobilization (Figure 5). pen as infiltration provides the necessary tension for scoring within the box.

In robotic hair extraction, a tensioner is used and provides a tight skin surface for precise entry of the robotic punch. This
FIGURE 6. Two-point two operator traction: the opposite hand of the operator provides upper traction while an assistant provides a point of counter-traction.

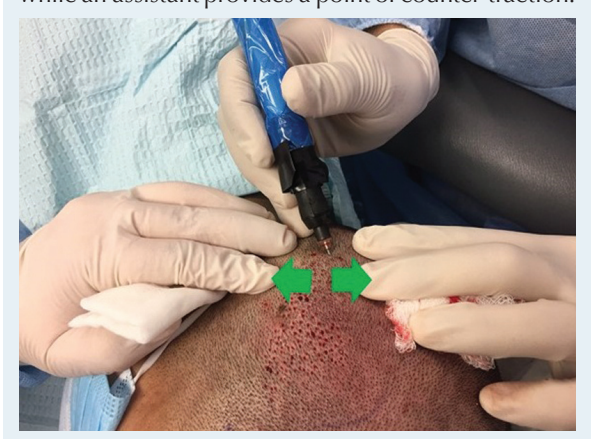

FIGURE 7. Three-point two operator traction: in addition to the operator's single-hand traction, two other hands of an assistant provide counter-traction which creates a 3-point traction.

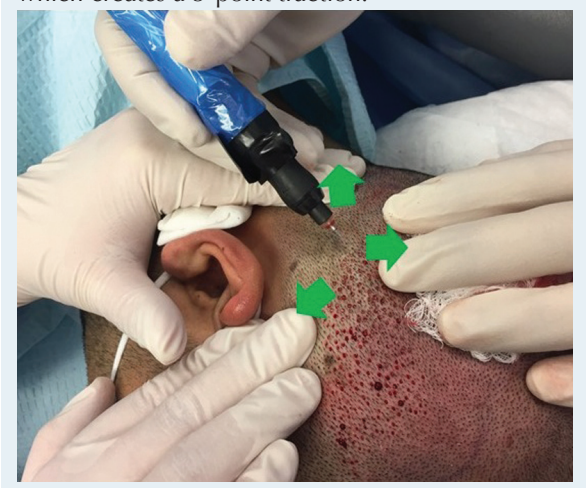

FIGURE 8. Tumescent effect: superficial infiltration of the dermis with saline provides one of the best traction of the scalp. The tension of the tumescent effect ligers only for a short time before the fluid dissipates into the interstitial space.

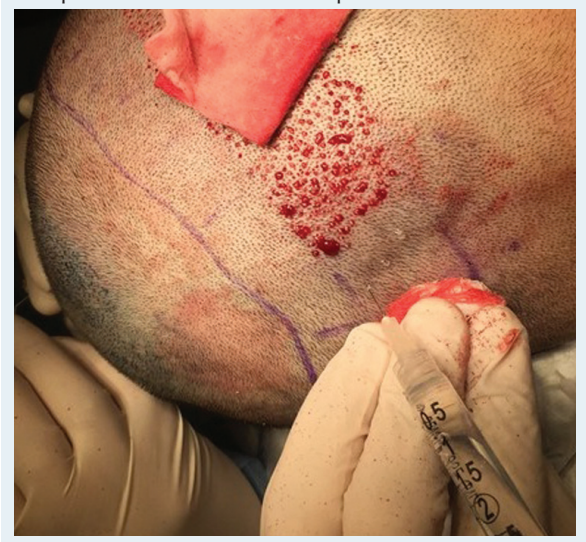

ganized and stepwise fashion, moving across the donor area. Small boxes should be outlined with a marking

FIGURE 9. Tensioner: as used in Robotic hair restoration, provides a 4 -point traction with even tension throughout the grid.

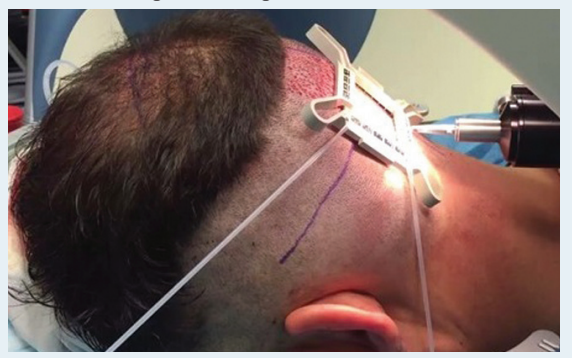


traction could be considered 4-point traction, which creates ideal and even tension throughout the target grid (Figure 9).

\section{PUNCH SETUP}

\section{Internal-External Angle (Int-Ext):}

Internal (below the skin) and external (out of the skin) angles of hair shafts are not always the same. The transection rate can increase if the surgeon only follows the external angles. Even in the same individual, the internal and external angles could be similar in some areas of the scalp while they may differ drastically in others (Figure 10). The internal angle of the follicles, or the average of the internal angles of individual hair shafts, in one FU is what needs to be followed
FIGURE 10. Internal-external hair angle is the difference between the external and internal average angle of follicles within a follicular unit.

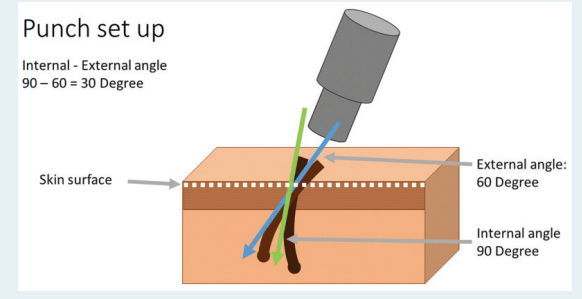

when scoring and punching the grafts. In comparing external and internal angles of hair follicles, the internal angles tend to be closer to the perpendicular axis of the skin. This means the operator should adjust the punch an-

gle according to the follicular internal angle at any area.

The internal angle is calculated in two ways:

1. There are some general rules for the Int-Ext angle relationship. Int-Ext angle varies depending on the extraction location within the donor region. The differences are usually less, or even nonexistent, in the temporal areas. In contrast, when approaching the more central areas of the permanent zone of the scalp, these angle differences become more pronounced. This difference in angle, based on the location of the donor hair, should be taken into consideration when scoring each graft.

2. The operator can use transected hair follicles to determine the angle change based on the transection location and depth. The punch should be angled more towards any surface of the graft that contains transected follicles.

\section{Punching steps}

1. Initial approach:

a. Motorized (off center). During motorized scoring, the initial approach of the punch is often off center when aligning the punch over the FUs (Figure 11). Subsequently, during the next step of the cutting maneuver,
FIGURE 11. Motorized punch insertion: a light downward pressure on the skin makes the punch centered while scoring the skin before punch advancement.

Initial approach:

Motorized

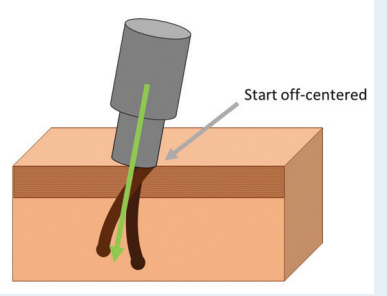
there is a light downward pressure on the skin, which results in the hair shaft(s) becoming centralized within the punch. It should be noted that despite of initial offcenter alignment of the punch, the final hair shafts on the grafts look centered in relation to the graft skin cap. Motorized punches are usually heavier, and it is more difficult to change the angles than with lighter manual punches. The off-centered approach helps to depress one edge of the skin where the punch touches first and creates an almost perpendicular surface for easier cutting of the skin with the punch edge.

b. Manual non-motorized (centered). When using manual or non-motorized punches, the hair shaft must be centered at the onset, as the punch is held perpendicular to the skin for the superficial scoring (Figure 12). The angle of the punch then changes during the follow-through to accommodate the exit angle and allow the shaft(s) to remain centralized as it is advanced to full depth (Figure 13).

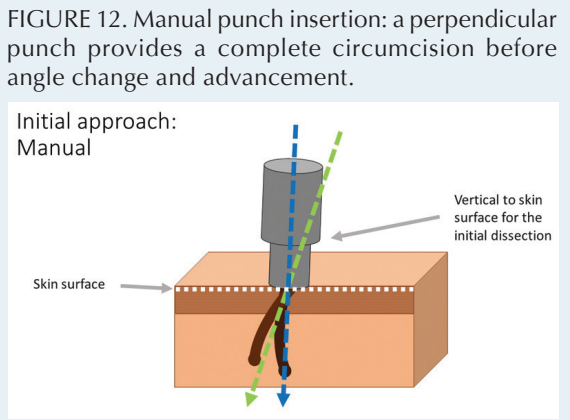

FIGURE 13. Angle change according to follicular unit internal axis is followed by advancement of punch after initial scoring.

Initial approach:

Manual

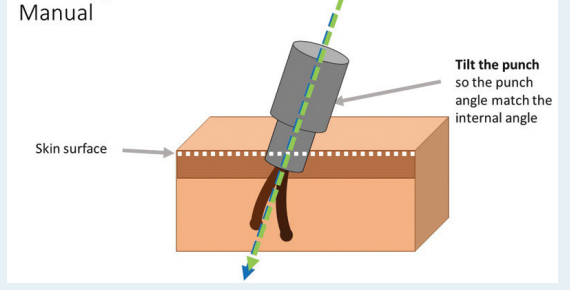

2. Pause-then-go technique. During motorized scoring, pause the punch tip after it touches the skin surface to allow for the engagement of the tip. This split-second pause enables the upper rim of the sharp punch to bury itself and disappear into the skin. In other words, the pause allows for the skin surface to be completely cut circumferentially before the punch is further advanced.

3. Advancing the punch. The final step is a quick advancement of the punch to complete the scoring of the graft through the anchoring system. This step should be done quickly and effortlessly, but only if the prior steps are done correctly. There should not be much resistance in advancing the punch since the toughest layers of scalp are located on the very top $2-3 \mathrm{~mm}$ of skin. A speedy advancement of the punch minimizes the contact time of the cutting edge alongside the follicles, which can translate into fewer follicles transected.

4. Depth control. The anchor system is limited to the upper $1-3 \mathrm{~mm}$ of the follicles in most cases. The anchor system should be completely cut to be able to extract the grafts effortlessly. When using a sharp punch, we have to minimize the depth of our cut to minimize the chance of transecting the follicles. Dull punches, as used in the ARTAS robotic approach, can be advanced to the entire length of follicles. Using a sharp punch, any splay in grafts or any angle change brings the cutting edge in touch with the follicles and can lead to partial or complete transection. Limiting the punch depth helps cut through the anchor system without unnecessarily increasing the risk of transecting the follicles. 


\section{PULLING SCORED GRAFTS}

1. Two-handed technique. The graft is removed in two steps:

- Step 1: fine forceps provide the initial gentle lift by gently grasping the graft at the epidermis level (Figure 14). These forceps may be serrated or not depending on the case. If the graft is not naturally elevated,

FIGURE 14. Step 1 of graft extraction: lifting the graft up

Graft extraction

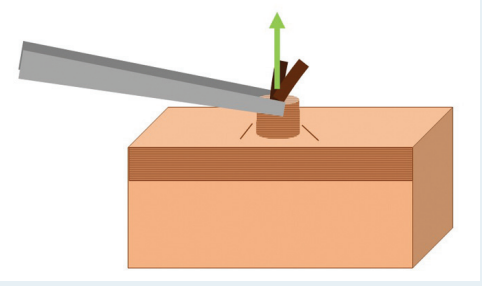

FIGURE 15. Step 2 of graft extraction

Graft extraction

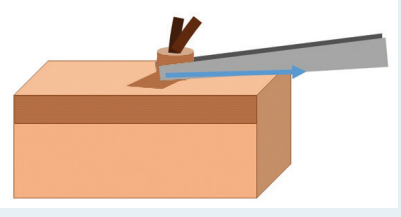

placing the forceps' tip into the canal may be required to elevate and retrieve the graft.

- $\quad$ Step 2: serrated forceps grab the graft below the first forceps (as low as possible along the graft), and pull in a direction that is perpendicular to the direction of the forceps serrations (Figure 15). This perpendicular force applied with the second forceps along with a deep grab will decrease the possibility of tethering.
If there is a problem pulling the grafts, the technician should promptly notify the surgeon. The most common reportable issues include:

- Tethering: resistance in pulling of the grafts. This implies the anchor system is not completely cut and the depth of scoring should be increased.

- Capping: pulling the epidermis and superficial dermis off the graft. Similar to tethering, capping may imply the anchor system is not cut and the graft is not completely freed.

- Partial or total transection: may indicate that the angle of the punch needs to be modified.

\section{TESTING GRAFT QUALITY}

To ensure optimal graft quality, it is mandatory to test or inspect the grafts that are initially extracted. Thereafter, periodic testing of grafts is necessary to safeguard quality. Maintenance of quality is done in a stepwise fashion:

1. One at a time. In the beginning, grafts should be extracted and inspected one at a time. During graft testing, information regarding proper punch depth, external/internal angles, and splay is gathered by the operator. Slight adjustments are made with each graft. This testing period, although time consuming, is a valuable and necessary step as it sets the parameters for a smooth rest of the day.

2. Three at a time. Once satisfied with the appearance of the grafts obtained one by one, the operator can increase the pace and score "three at a time," inspecting every third graft, then fine-tuning while moving faster until the normal speed of the extraction is reached.

3. Testing while extracting with no interruption. It is advisable that a technician periodically check the last few grafts scored, especially in a zone of transition. This is particularly important if there is a significant distance (of greater than 2 inches), between the surgeon and the extracting technician. Grafts should be displayed to the surgeon for quick viewing without disruption of the scoring process.

Periodic testing helps detect any subtle changes that may occur from one area to another. Testing should be done expeditiously, particularly if the surgeon notes any signs of poor extraction. Signs of poor scoring are:

a. Grafts not elevated properly or appear depressed (Figure 16).

b. Grafts are rotated and skin cap is not parallel to the scalp (Figure 17).

c. Grafts disappear after scoring (missing grafts)

(Figure 18).

\section{Signs of good scoring}

The surgeon should realize when the scoring and extraction are going well. Recognizing the following clues of a well-scored graft will minimize unnecessary graft testing and reduce the overall technician pulling time. Positive signs include (Figure 19):

1. Grafts should be $0.5-1 \mathrm{~mm}$ elevated. This means the anchor system is properly disengaged thus freeing the graft to allow for easy removal.

2. Graft skin cap is parallel to the scalp. This usually indicates that the graft is properly scored and less likely to have a partial or complete transection.

3. Effortless pulling of grafts by the technicians. Effortless pulling denotes proper scoring with correct depth. If there is any tethering, the surgeon should increase the
(6. Depressed graft: a sign of poor Signs of poor extraction

FIGURE 17. Rotated graft

Signs of poor extraction

Grafts are rotated and
the cap is not parallel to the skin surface

FIGURE 18. Missing graft

Signs of poor extraction

FIGURE 19. Signs of good graft extraction: 1) elevated graft, 2) parallel skin cap, 3) effortless

Signs of good extraction

1. Elevated grafts:

The anchor system is

2. The skin cap is

surface

3. Extraction and

effortless 
depth gradually to find the minimal depth required that will free the grafts from their anchoring points.

\section{CONCLUSION}

Within the past decade, we have witnessed a revolution in the field of hair restoration with the emergence of more efficient methods of performing FUE. As the field of FUE hair restoration improves, there is a high demand for standardizing the process. The dynamics involved with FUE transplantation techniques used in our practice are based on the techniques that have evolved over the past decade. Although some of these concepts have evolved through our work, many of them are based on collective information gathered from many great minds in the field of hair restoration. This information was collected from a variety of sources including publications, meetings, workshops, and even hallway discussions.
These changes have been so far reaching that our methods of performing the procedures have drastically changed and reached the point where what is performed in our practice looks like a completely different procedure when compared with the methods used five to seven years ago. This transition is not limited to our practice; we have witnessed a similar trend in most leading hair restoration clinics around the globe.

Hair transplant surgeons can make their results more predictable using standard techniques. Although there are currently other methods of FUE available, the alternative and parallel methods are becoming similar as our understanding of the process improves. Understanding the dynamics involved with FUE allows hair transplant surgeons to create reproducible results and also helps patients achieve their goals with every procedure.

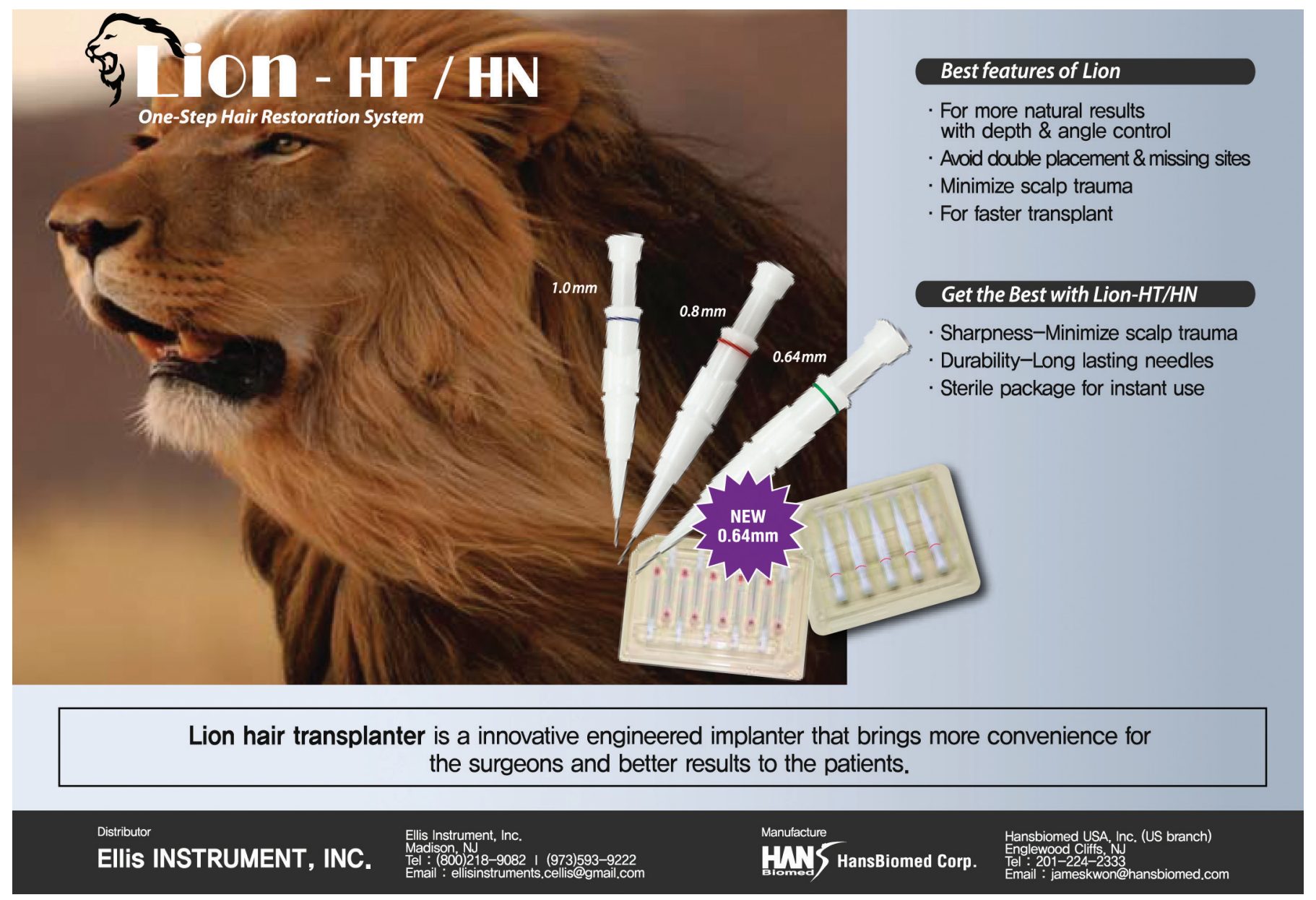

